\title{
Detection of evaluation bias caused by genomic preselection
}

\author{
A.-M. Tyrisevä, ${ }^{* 1}$ E. A. Mäntysaari, ${ }^{\star}$ J. Jakobsen,† G. P. Aamand, $\ddagger$ J. Dürr,§ W. F. Fikse, $\#^{2}$ and M. H. Lidauer* \\ *Natural Resources Institute Finland (Luke), Green Technology, Biometrical Genetics, 31600 Jokioinen, Finland \\ †Norwegian Association of Sheep and Goat Breeders, 1431 Ås, Norway \\ ‡NAV Nordic Cattle Genetic Evaluation, 8200 Aarhus, Denmark \\ §Council on Dairy Cattle Breeding, Bowie, MD 20716 \\ \#Department of Animal Breeding and Genetics, Swedish University of Agricultural Sciences, 75007 Uppsala, Sweden
}

\begin{abstract}
The aim of this simulation study was to investigate whether it is possible to detect the effect of genomic preselection on Mendelian sampling (MS) means or variances obtained by the MS validation test. Genomic preselection of bull calves is 1 additional potential source of bias in international evaluations unless adequately accounted for in national evaluations. Selection creates no bias in traditional breeding value evaluation if the data of all animals are included. However, this is not the case with genomic preselection, as it excludes culled bulls. Genomic breeding values become biased if calculated using a multistep procedure instead of, for example, a single-step method. Currently, about $60 \%$ of the countries participating in international bull evaluations have already adopted genomic selection in their breeding schemes. The data sent for multiple across-country evaluation can, therefore, be very heterogeneous, and a proper validation method is needed to ensure a fair comparison of the bulls included in international genetic evaluations. To study the effect of genomic preselection, we generated a total of 50 replicates under control and genomic preselection schemes using the structures of the real data and pedigree from a medium-size cow population. A genetic trend of $15 \%$ of the genetic standard deviation was created for both schemes. In carrying out the analyses, we used 2 different heritabilities: 0.25 and 0.10 . From the start of genomic preselection, all bulls were genomically preselected. Their MS deviations were inflated with a value corresponding to selection of the best $10 \%$ of genomically tested bull calves. For cows, the MS deviations were unaltered. The results revealed a clear underestimation
\end{abstract}

Received July 19, 2017.

Accepted December 10, 2017.

${ }^{1}$ Corresponding author: anna-maria.tyriseva@luke.fi

${ }^{2}$ Current address: Växa Sverige, 75105 Uppsala, Sweden. of bulls' breeding values (BV) after genomic preselection started, as well as a notable deviation from zero both in true and estimated MS means. The software developed recently for the MS validation test already produces yearly MS means, and they can be used to devise an appropriate test. Mean squared true MS of genomically preselected bulls was clearly inflated. After correcting for the simulated preselection bias, the true genetic variance was smaller than the parametric value used to simulate BV, and also below the variance based on the estimated BV. Based on this study, the lower the trait's heritability, the stronger the bias in estimated BV and MS means and variances. Daughters of genomically preselected bulls had higher true and estimated BV compared with the control scheme and only slightly elevated MS means, but no effect on genetic variances was observed.

Key words: genomic preselection, Mendelian sampling, evaluation bias, data validation

\section{INTRODUCTION}

Unbiased comparison of dairy bulls among countries ensures efficient genetic progress of herds for dairy farmers and fair trade for breeding companies selling the best-ranking bulls on the global market. The bulls included in Interbull international evaluations receive EBV in each participating country's own unit, scale, and base, which enables comparisons of national and foreign sires. Interbull international evaluations employ multiple across-country evaluations (MACE), with national evaluation results, such as breeding values (BV) and transmitting abilities (TA), as input. The MACE has been found to be sensitive to the quality of national evaluation models and, consequently, to the BV or TA that countries provide for inclusion in Interbull international sire evaluations (e.g., Ducrocq et al., 2003). As observed in many studies, MACE is exposed to and affected by biased genetic trends and biased genetic variance trends in the national evaluations. Bulls from 
countries that overestimate their genetic trends, as well as bulls from birth year classes with inflated genetic variances, gain unfair advantage (Weigel et al., 1996; Van Doormaal et al., 1999; Gengler et al., 2000; Miglior et al., 2002; Ducrocq et al., 2003).

The era of genomic selection has given rise to a new source of bias: namely, bias due to genomic preselection of young bulls unless adequately accounted for in national genetic evaluations. The $\mathrm{BV}$ of each animal is estimated by mixed model equations (MME), including the parental average, the animal's own yield deviation, and the contribution of its offspring. Sorensen and Kennedy (1984) found that selection caused no bias in traditional genetic evaluation, provided that the data and pedigree of all animals were included; however, that is not the case with genomic preselection if data on culled bulls are excluded. Genomic enhanced breeding values (GEBV) also become biased if calculated using a multistep procedure (Patry and Ducrocq, 2011b; Vitezica et al., 2011). This procedure first involves traditional BV estimation to obtain pseudo-observations for the genotyped animals, utilizing their phenotyped relatives, after which these pseudo-observations are combined with marker information to determine the genotyped animals' GEBV (e.g., VanRaden, 2008; Hayes et al., 2009). The pseudo-observations are no longer a random sample of Mendelian sampling (MS) and the effect of selection cannot be accounted for, as only a subset of the available data are used to obtain GEBV (Ducrocq and Liu, 2009; Vitezica et al., 2011).

To solve the inherent source of bias introduced by genomic preselection, researchers have worked hard to reap the benefits of genomics while at the same time overcoming the challenges it brings. Indeed, new methods have been developed that are unbiased or at least reduce bias. For instance, one of the suggested approaches transforms GEBV into weighted deregressed performances, which are then used together with the original phenotypes in BLUP evaluations, allowing the inclusion of culled bulls (Ducrocq and Liu, 2009; Patry and Ducrocq, 2011a). The most elegant option, although computationally more demanding, is a singlestep method (e.g., Aguilar et al., 2010; Christensen and Lund, 2010), which is an extension of traditional MME. It adds a new source of information, genotypes, to the traditional data from the animal itself, its parents, and its offspring. For this method, genotyped as well as nongenotyped animals are included in the model, and unbiasedness is achieved by including culled bulls. Development efforts to ease the computational burden of the single-step method are ongoing in several countries, and various solutions have already been suggested (e.g., Misztal et al., 2014; Taskinen et al., 2017).
Currently, 18 of the 31 countries $(60 \%)$ participating in international bull evaluations have already adopted genomic selection in their breeding schemes: some of them only for production traits, some for a wider variety of traits. So far, only 2 of the 18 countries have implemented the single-step approach, whereas the majority rely on the original multistep approach (Interbull, 2017). This makes the data sent for MACE from different countries very heterogeneous and creates a real risk of bias due to genomic preselection.

In a simulation study with 3 countries, Patry et al. (2013) observed that genomically preselected bulls from countries that send biased data for international evaluations were penalized both for the biased trait and for correlated traits of other countries. In the context of $\mathrm{MACE}$, the correlated trait, although the same biological trait, is treated as a different trait by various countries, allowing less-than-unity genetic correlation. The penalizing effect was to some extent also transmitted to the relatives of the selected bulls. Patry et al. (2013) further showed that failure to account for genomic preselection in national evaluations had a more severe effect than provision of incomplete (excluding culled animals) but unbiased data for international evaluations. The current heterogeneous situation among participating countries makes it very difficult to predict the overall effects of genomic preselection on the accuracy of MACE. VanRaden and Wright (2013) pointed out that bias is likely to increase in the near future, as elite young bulls are increasingly mated to elite genotyped cows or to cows with many good sons. Therefore, methods to account for genomic selection in national evaluations are needed, as well as for proper validation methods to detect possibly biased EBV.

A new test to validate the consistency of MS variance was recently developed and has been approved among the compulsory validation tests for countries participating in international sire evaluations (Tyrisevä et al., 2018). A tailored program for conducting the analyses is also available. The validation procedure estimates within-year genetic variances utilizing information on animals' MS values and also tests for a possible trend and outliers in the estimated variances.

The aim of our simulation study was to establish whether it is possible to use the new MS validation test to detect the bias caused by genomic preselection of young bulls either from the estimates of within-year MS means or variances. Theoretically, the variance of true breeding values of selected individuals is expected to decrease as a result of genomic preselection (Falconer and Mackay, 1996); further, the mean of MS deviations for selected individuals is expected to differ from zero (Patry and Ducrocq, 2011b). 
MATERIALS AND METHODS

\section{Simulation Method}

To create data sets with a desired genetic trend, we used a new simulation method described for the first time by Tyrisevä et al. (2013). In this method, the dependent variable in the original data is first replaced with pseudo-observations having the desired annual trend, and these pseudo-observations are then used to predict pseudo-BV expressing the genetic trend. The pseudo-BV become synchronized with the parent and progeny averages and the expected yearly means of BV. Together with the pedigree data, these pseudo-BV are then used to simulate true BV for each animal. Hence, the selection differential caused by the genetic trend will be transmitted in the MS part of the animal's BV. To ensure that the MS part of the parental BV is not regressed toward yearly means, the observations of parent animals are set as missing in the first step.

In the next step, true breeding values (TBV) are generated recursively generation by generation so that the BV of each animal consists of the parental mean, the random MS sampled from the normal distribution, and the MS created in the first step and carrying the genetic trend. Finally, the other random effects in the model, including residual effects, are generated and then summed to form observations.

Normally, MS variance $\left(\sigma_{\varphi}^{2}\right)$ can be expressed as $\sigma_{\varphi}^{2}=d_{j j} \sigma_{u}^{2}$, where $d_{j j}$ is the diagonal of an animal $j$ in the matrix $\mathbf{D}$ for Mendelian sampling in the decomposition of $\mathbf{A}=\mathbf{L D L}^{\mathbf{T}}$, where $\mathbf{L}$ is the lower unitriangular transition matrix, and $\sigma_{u}^{2}$ is the additive genetic variance. However, this property $\left(\sigma_{\varphi}^{2}=d_{j j} \sigma_{u}^{2}\right)$ no longer applies when the expectation of MS differs from zero. Mendelian sampling variance increases with the variance of the MS created in the first step, also leading to inflated BV variance. To avoid this, the random MS values were corrected when TBV were generated. The variance correction was carried out as

$$
\sigma_{\varphi}^{2}=(1-k) d_{j j} \sigma_{u}^{2}
$$

According to the standard formula by Falconer and Mackay (1996), $k=i(i-x)$, where $i$ is the selection intensity and $x$ is the deviation of the truncation point from the mean in standard deviation units. The selection intensity can be further formulated as $i=E[\varphi] / \sigma_{\varphi}$, where $E[\varphi]$ is the expected MS value. As $(1-k)$ is an exponential function of $i$, a satisfactory approximation can be obtained by a linear fit on its logarithmic value. A good fit was obtained with the formula

$$
(1-k)_{j}=\operatorname{Exp}\left(-1.18969|i|+0.10805 i^{2}\right),
$$

where $|i|$ is the absolute value of $i$.

\section{Data Used for Simulations}

A field data set of 754,600 Danish Holstein cows from 2,000 herds was sampled for the simulations. The time interval covered $20 \mathrm{yr}$, and the pedigree data comprised 1.2 million animals. Only the herd and pedigree structure were retained from the original data, and an artificial trait was simulated. One record was generated for each cow. The model applied in both steps included a fixed herd effect as well as random additive genetic and residual effects. To generate the pseudo-BV, we used a heritability of 0.05 to ensure that the base level of the MS means in each birth year class remained close to zero. In simulating the final data sets, we applied 1 of 2 heritabilities: 0.10 or 0.25 . A genetic variance of 1,650 was assumed for all analyses.

\section{Study Design}

First, we created observations with an annual trend of $15 \%$ of the genetic standard deviation to be used in solving pseudo-BV and in calculating MS deviations. This was done only once and used for all data replicates thereafter. Second, we created control and genomic preselection (GPS) schemes with 50 data replicates each. The same seeds were used for the replicates in both schemes. To mimic a genomic preselection in the GPS scheme, we assumed all bulls from the birth year class 2000 onwards to be genomically preselected. The MS values obtained from the pseudo-BV generation step for these bulls were increased with a MS+ value calculated as

$$
M S+=\sigma_{\varphi} \times i \times r=\sqrt{1,650 / 2} \times 1.755 \times 0.60=31,
$$

where $r$ describes the accuracy of the predictions. The MS+ value corresponds to selection of the best $10 \%$ of genomically tested bull calves. The MS values of cows were unaltered.

\section{Analyses}

We calculated TBV and EBV under both schemes and used the results to obtain within-year means of TBV and EBV. The simulations were carried out with MiX99 software (MiX99 Development Team, 2015), modified to generate TBV using the generated MS distribution with a nonzero expectation. We further determined the bias (EBV minus TBV) as well as the 
ranking of top $10 \%$ of bulls from birth years around the start of GPS. The generated TBV and EBV and reliabilities of TBV and EBV were used to calculate within-year true genetic variance (TGV; i.e., mean squared TMS) and estimated genetic variance (EGV) with the MS variance validation program (Tyrisevä et al., 2018), which was also used to obtain within-year true and estimated MS (TMS, EMS) means. Under the GPS scheme, TGV was additionally calculated by correcting for the inflation of variance $\left(31^{2}=961\right)$ due to the preselection bias $\left(\mathbf{T G V} \mathbf{V}^{*}\right)$. The approximated EBV reliabilities were computed by the method of Misztal and Wiggans (1988), implemented in the Apax99 software (MiX99 Development Team, 2015). The reliabilities of TBV were 1.0.

\section{RESULTS AND DISCUSSION}

\section{Genetic Trend and the Effect of Genomic Preselection on BV}

Under the control scheme, the pseudo- and true BV for bulls displayed very similar genetic trends (Table 1 ), with the same yearly genetic progress (7.1 units/year). Further, the within-year means of EBV estimated from the generated data sets followed the underlying TBV very closely, with a genetic progress of 7.0 units/year. The adoption of GPS in 2000 produced a steep increase in the level of TBV (Table 1, Figure 1). Genetic progress for TBV was clearly higher under the GPS scheme than the control scheme: 9.9 units/year; yet, only part of this genetic progress, 8.5 units/year, was detected when EBV were predicted from the generated data sets. As a result of GPS being unaccounted for in the evaluations, bulls' yearly EBV means began to increase before GPS started, as the superiority of GPS bulls was transferred to their parents' EBV. This becomes evident when EBV means of parents of the bulls are studied (Table 1); they were clearly overestimated and notably biased compared with the control scheme, even before start of GPS.

The small detected bias under the control scheme in bulls was possibly due to the unaccounted-for inbreeding effects (Table 1). In contrast, in the GPS scheme the bias grew steadily until the start of GPS, after

Table 1. Within-year means of pseudo, true (TBV), and estimated (EBV) breeding values, as well as average biases expressed as EBV - TBV ${ }^{1}$

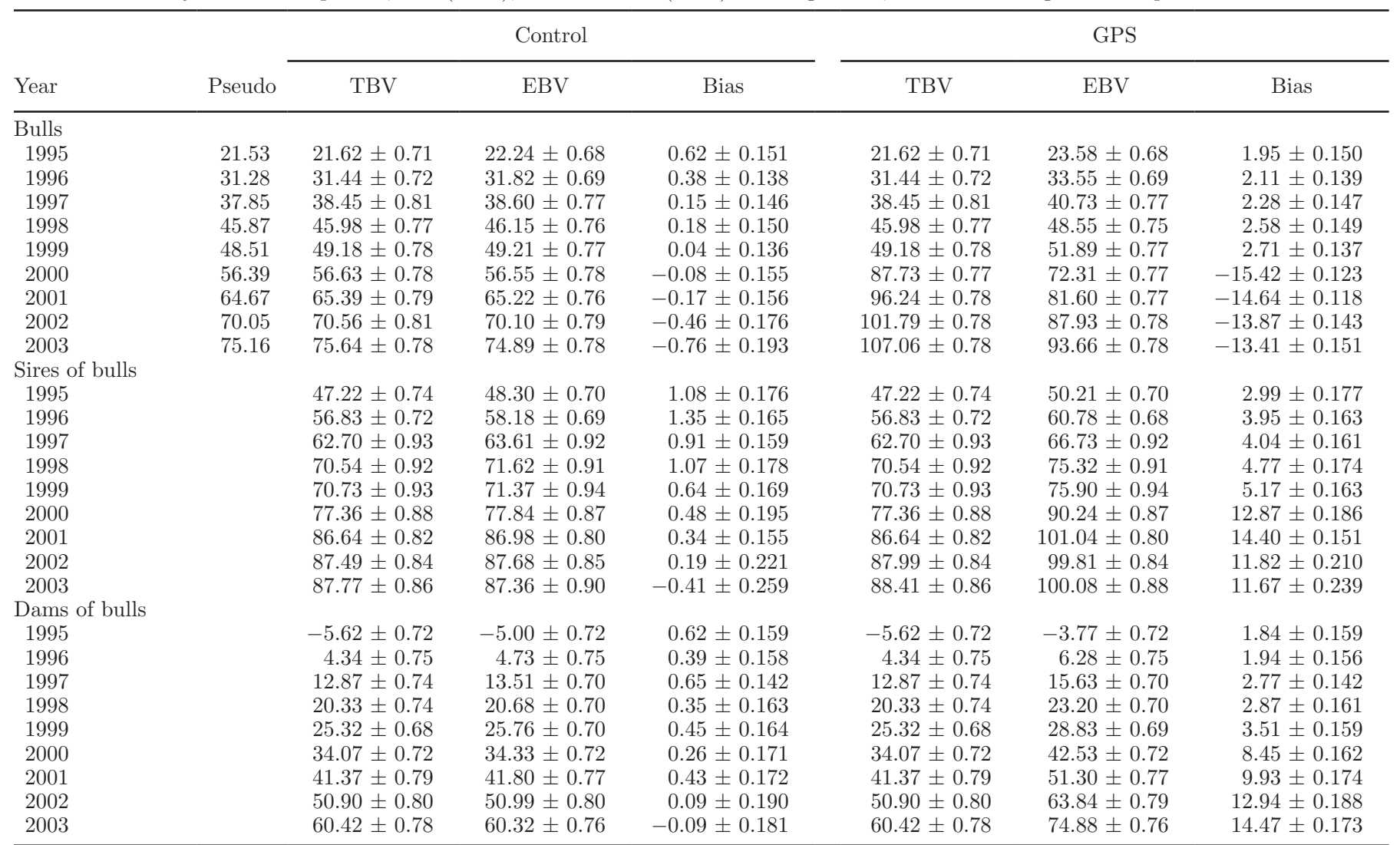

${ }^{1}$ For the means of TBV, EBV, and the bias, the SE are also shown. Means are shown for bulls and their sires and dams. The year refers to the birth year class of bulls; that is, for sires and dams the year is their son's birth year. The means of pseudo breeding values were derived from one data set, whereas the results from the control and genomic preselection (GPS) schemes were averaged over 50 replicates. GPS started in 2000. A heritability of 0.25 was used. 
which a steep increase occurred due to underestimated $\mathrm{BV}$ of bulls. The underestimation of genetic progress in the GPS scheme was even more pronounced when EBV were predicted from data sets with lower heritability (results not shown).
A slight increase in the yearly means of cows' TBV and EBV could also be detected 2 yr after the start of GPS of bulls, when the first GPS bulls became sires (Figure 1). The results are in a good accordance with those reported by Patry and Ducrocq (2011b).

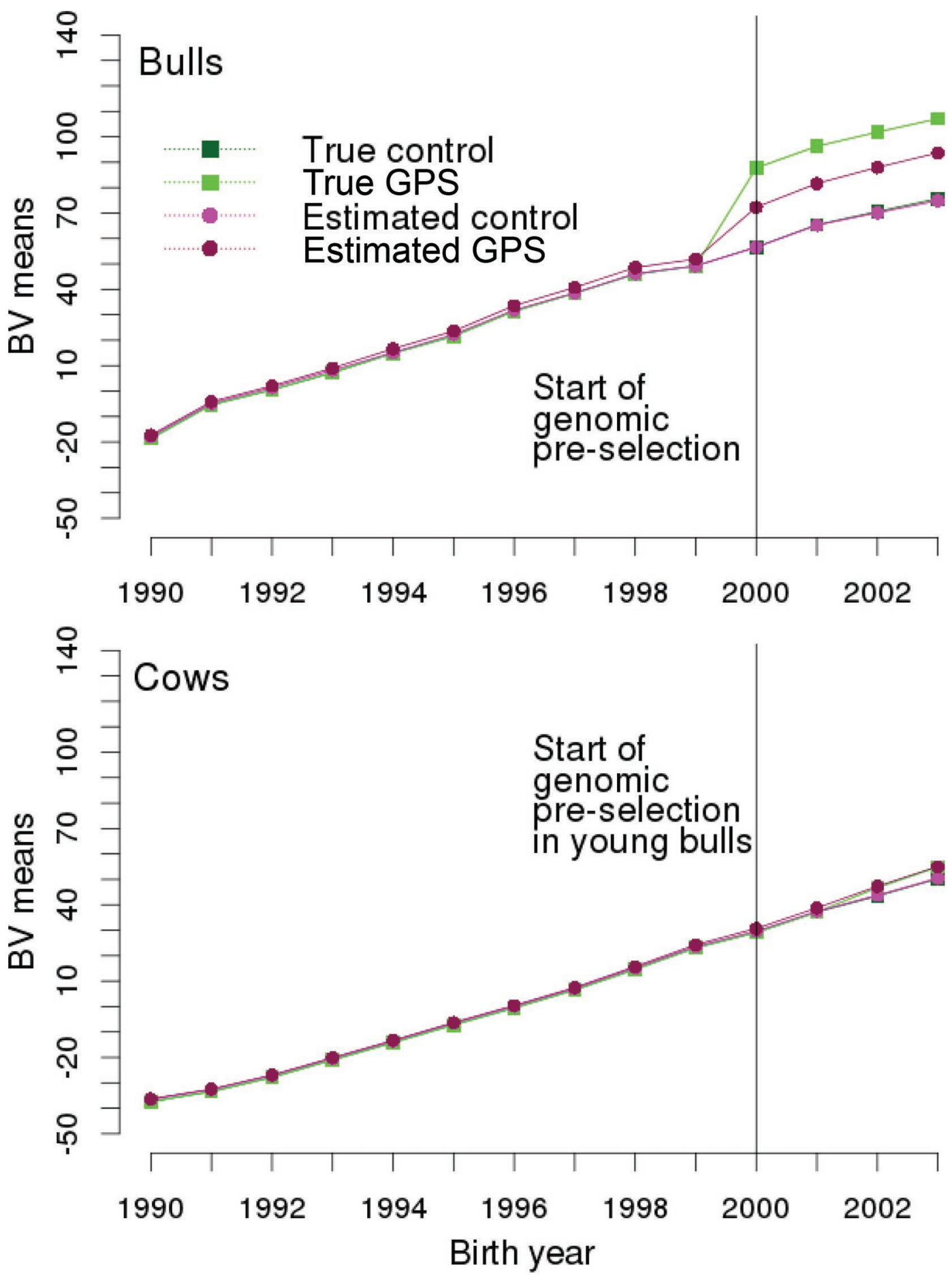

Figure 1. Within-year means of true and estimated breeding values (BV) for bulls and cows from the control and genomic preselection (GPS) schemes. Means were averaged over 50 replicates. A heritability of 0.25 was used. Color version available online. 


\section{Effect of Genomic Preselection on MS Means}

Both TMS and EMS means for bulls were very close to zero under the control scheme (Figure 2). Under the GPS scheme, the TMS means were identical with those from the control scheme until the start of GPS, after which an expected increase of +31 was observed. The EMS means deviated clearly from zero following the start of GPS. However, the increase was only around $1 / 4$ of that seen in the TMS means, which corresponded to $1 / 2$ of the observed increase in bulls' EBV due to
GPS. For cows, both the TMS and EMS means were practically zero under the 2 schemes, although a minor effect on EMS means could be detected after the adoption of GPS of bulls (Figure 2). Patry and Ducrocq (2011b) reported similar results from their simulations.

When the analyses were repeated using the same yearly genetic trend but with lower heritability (0.10), the EMS means still deviated from zero after GPS started but the effect was smaller compared with analyses with higher heritability: only $1 / 6$ of that found for TMS means. This is a logical outcome, as the observed
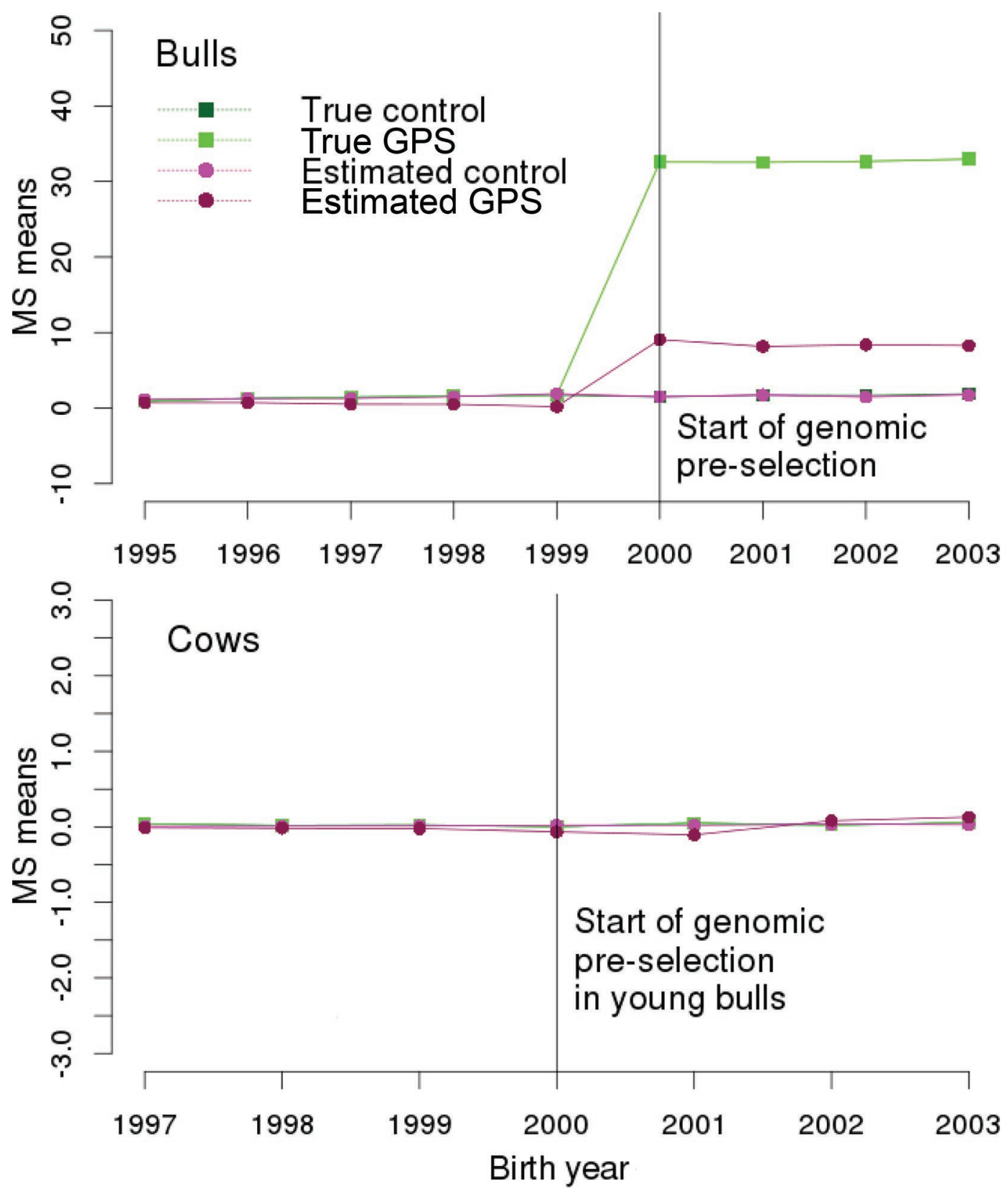

Figure 2. Within-year means of true and estimated Mendelian sampling (MS) terms for bulls and cows from the control and genomic preselection (GPS) schemes. Means were averaged over 50 replicates. A heritability of 0.25 was used. Color version available online. 
increase in bulls' EBV due to GPS was smaller when a heritability of 0.10 instead of 0.25 was applied. Thus, the bias was larger compared with the heritability 0.25 setting.

\section{Effect of Genomic Preselection on Genetic Variances}

For cows, the TGV and EGV under both schemes were virtually identical with the variance used for BV simulations (Figure 3). The similarity of genetic variance from the control scheme with that applied in the simulations implies that the formula used for variance correction had a good fit.

On the whole, the level of genetic variance was a bit lower for bulls than for cows (Figure 3). With 0.25 heritability, the EGV from the control scheme, averaged over 50 replicates and all the studied years, was 1,509. At least 2 reasons for this can be found. The overall level of EMS means for bulls deviated slightly from zero compared with cows (Figure 2); this was further reflected in the level of estimated genetic variance (E. A. Mäntysaari, A.-M. Tyrisevä, and I. Strandén, Natu-
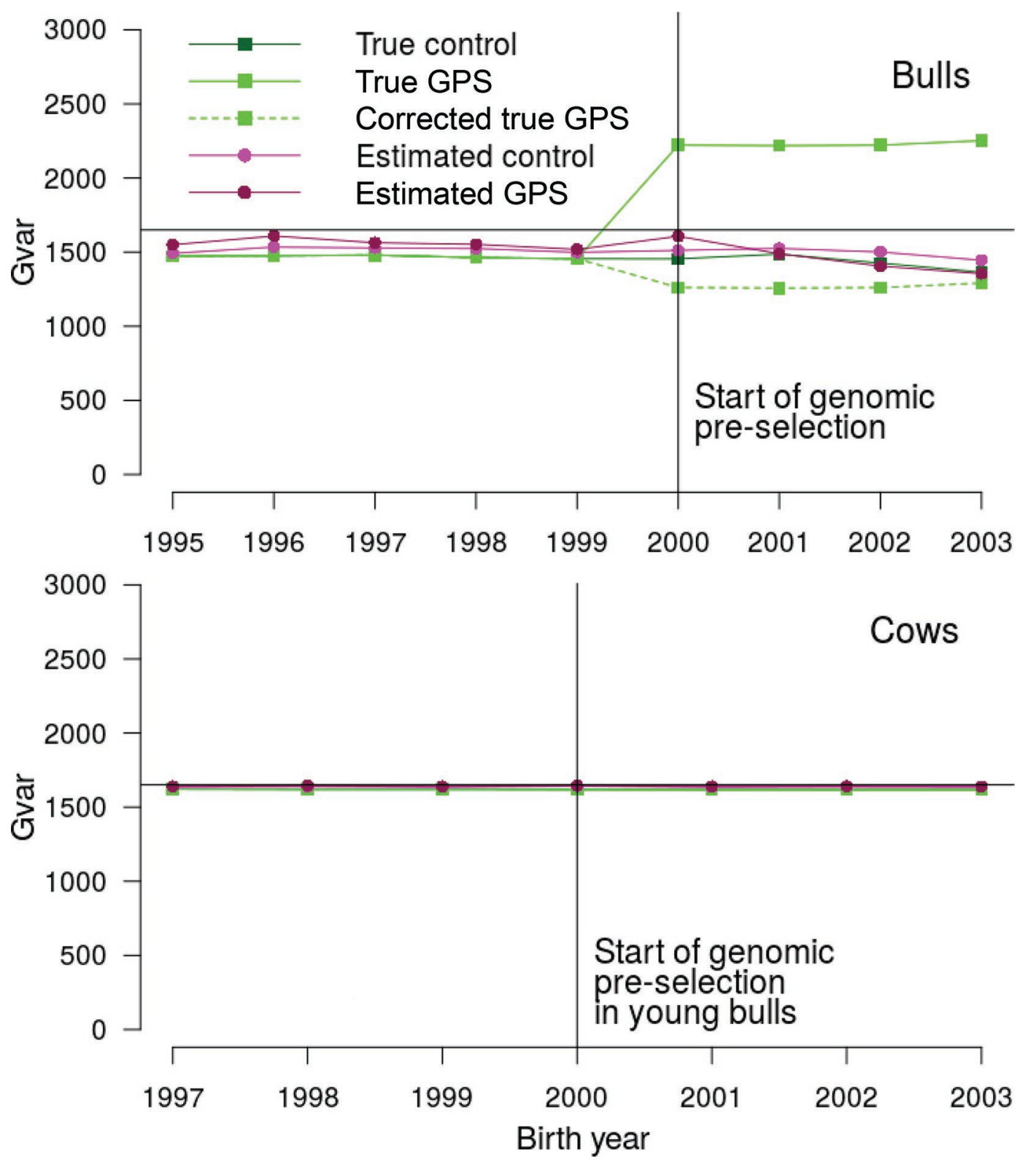

Figure 3. Within-year means of true and estimated genetic variances (Gvar) for bulls and cows from control and genomic preselection (GPS) schemes. For the GPS scheme, both original true values and those corrected for the simulated preselection bias are shown. Means were averaged over 50 replicates. A heritability of 0.25 was used. Variance used for breeding value simulations is marked with a solid black line. Color version available online. 
ral Resources Institute Finland, Jokioinen, Finland, unpublished data). Another reason for the difference between cows and bulls is that selection is mainly carried out through bulls, which reduces variance of their TBV.

For bulls, a slight descending trend was observed in TGV $(-0.6 \%)$ and EGV $(-0.3 \%)$ under the control scheme. Inbreeding may be one reason for this, as it can cause a decrease in genetic variance as found by Tyrisevä et al., (2018). Immediately when GPS started, a distinct increase occurred in TGV (Figure 3) as a response to the elevated TMS means. After GPS, the level of TGV remained relatively steady throughout the studied time period. However, when TGV was corrected for the simulated preselection bias (equal to $31^{2}$, Figure 3 ) the adoption of GPS created a clear decline in $\mathrm{TGV}^{*}$ for preselected bulls. The $\mathrm{TGV}^{*}$ was the lowest of all variances shown, consistent with the theoretical expectation (Falconer and Mackay, 1996). The effect of GPS on EGV was reverse to the TGV and clearly smaller in magnitude. However, when compared with the TGV*, EGV under the GPS scheme was higher due to the inflated EMS of genomically preselected bulls (Figures 2 and 3). Before the adoption of GPS, the level of EGV between the control and GPS schemes differed, indicating the same type of leverage effect as seen in the means of bulls' EBV. When GPS started, the level of EGV first increased slightly and then slowly decreased, reaching the level of $\mathrm{TGV}^{*}$ in the fourth year under GPS. The increase in variance at the start of GPS reflected an inflated MS variance. This is because the expectations of MS were greater than the parental averages, whereas the EMS values were regressed toward parental averages due to below 1.0 accuracy of bull EBV. In the years following GPS adoption, the difference between expectations of MS and parental averages narrowed, as the genetic trend of the parents grew stronger due to an increased bias in their EBV (Table 1). This, in turn, reduced the inflation of EMS variance, and by the year 2003 resulted in estimates close to $\mathrm{TGV}^{*}$.

By and large, all consequences of GPS on EGV were small for bulls and hard to disentangle from other sources contributing to trends in EGV. The descending trend for EGV in the GPS scheme and with 0.25 heritability was $-1.5 \%$. The decreasing trend reached a level of $-2 \%$ for 14 replicates out of 50 (28\%), whereas in the control scheme only 1 replicate out of 50 attained that level. The $\pm 2 \%$ trend in EGV is the threshold of acceptance suggested in the MS variance test (Tyrisevä et al., 2018).

Decreasing the heritability from 0.25 to 0.10 influenced the overall level of genetic variance for bulls to some extent. The average EGV from the control scheme was 1,565 . Due to GPS, the scenario with 0.10 heritability produced similar patterns as with 0.25 heritability, although smaller in magnitude. The descending trend in EGV was only $-0.8 \%(-0.1 \%$ under the control scheme), and only 2 out of 50 replicates reached the $-2 \%$ level (none in the control scheme). These results indicate that the higher the heritability, the stronger the decreasing trend in genetic variance due to unaccounted GPS. It must be kept in mind, however, that the genetic trend simulated in our study ( $15 \%$ of genetic SD) was identical for both heritability settings. Even though it enabled an identical and comparable setting, such a high genetic trend for low heritability traits may be unrealistic in practice.

\section{Effect of Genomic Preselection on Top-Ranking Bulls}

Differences in the ranking of bulls based on their TBV (TRANK) and EBV (ERANK) under the control scheme were minor, whereas larger differences were observed under the GPS scheme (Table 2). A comparison of ERANK with TRANK in the GPS scheme showed that birth year classes 2001 to 2002 were underrepresented. With TRANK, birth year classes before 2000 provided $34.5 \%$ of top-ranking bulls in the control scheme and $12.3 \%$ under the GPS scheme. With ERANK, their proportion was almost the same as with TRANK in the control scheme (34 vs. $34.5 \%$ ), whereas in the GPS scheme it clearly grew too large (14.9 vs. $12.3 \%$ ). Hence, the difference in EBV means for bulls born before and after GPS was underestimated, resulting in a ranking with too few bulls from the underestimated birth year classes of the youngest bulls and too many from the overestimated classes of the oldest bulls, in accordance with the findings in the EBV means of parents and their sons in Table 1 . The results for the trait with 0.10 heritability were very similar, but ERANK under the GPS scheme gave an even greater proportion of top bulls born before GPS. This was probably due to more severe underestimation of the genetic trend than with the higher heritability trait.

\section{CONCLUSIONS}

Our results revealed a clear underestimation of bulls' EBV after the start of GPS accompanied with an overestimation of their parents' EBV. This was further mirrored to the ranking of bulls. Bulls born after the adoption of GPS were underrepresented in the top ranks if ranking was based on EBV. Further, we detected a notable deviation from zero both in TMS and EMS means of bulls. The software developed recently for the MS validation test to validate the consistency of MS 
Table 2. Distribution of the top $10 \%$ of the bulls into birth year classes 1997 to 2002 according to true (TBV) and estimated (EBV) breeding values from the control and genomic preselection (GPS) schemes ${ }^{1}$

\begin{tabular}{|c|c|c|c|c|}
\hline \multirow[b]{2}{*}{ Year } & \multicolumn{2}{|c|}{ Control } & \multicolumn{2}{|c|}{ GPS } \\
\hline & TBV & EBV & TBV & EBV \\
\hline 2002 & 24.5 & 24.2 & 33.6 & 31.1 \\
\hline 2001 & 26.8 & 27.4 & 36.3 & 35.3 \\
\hline 2000 & 14.3 & 14.4 & 17.8 & 18.6 \\
\hline 1999 & 12.5 & 12.5 & 4.6 & 5.7 \\
\hline 1998 & 14.2 & 14.7 & 5.3 & 6.6 \\
\hline 1997 & 7.8 & 6.8 & 2.4 & 2.6 \\
\hline
\end{tabular}

${ }^{1}$ Results were averaged over 50 replicates. GPS started in 2000. A heritability of 0.25 was used.

variance of the national evaluation models of Interbull member countries already produces yearly MS means that, in principle, can be used to devise a test to detect bias in MS means. However, the documentation of the properties of such a test will be required. The adoption of GPS created a modest decrease in EGV, which was the outcome of 2 effects: a decrease in genetic variance due to genomic preselection and an inflation of EGV due to biased EMS. Our results indicate that the lower the heritability for the trait, the stronger the bias in EBV and EMS means and EGV. Hardly any effect of GPS of young bulls was detected in their daughters, but the situation is likely to change in the near future as genomic testing of females becomes more popular. The pedigree structure used in this study was from the progeny testing scheme. In fact, the effects of GPS on EBV and EMS means and EGV would probably have been greater had we applied a pedigree more closely resembling that of the real GPS scheme.

\section{ACKNOWLEDGMENTS}

This study was conducted in cooperation with the Natural Resources Institute Finland (Luke, Jokioinen, Finland), the Interbull Centre (Uppsala, Sweden), the Swedish University of Agricultural Sciences SLU (Uppsala, Sweden), and NAV Nordic Cattle Genetic Evaluation (Aarhus, Denmark), all of which are acknowledged for their financial support. We also thank NAV for providing the Nordic Holstein test-day data for this study. The anonymous reviewers are thanked for their excellent contribution in improving the manuscript.

\section{REFERENCES}

Aguilar, I., I. Misztal, D. L. Johnson, A. Legarra, S. Tsuruta, and T. J. Lawlor. 2010. A unified approach to utilize phenotypic, full pedigree, and genomic information for genetic evaluation of Holstein final score. J. Dairy Sci. 93:743-752.
Christensen, O. F., and M. S. Lund. 2010. Genomic prediction when some animals are not genotyped. Genet. Sel. Evol. 42:2.

Ducrocq, V., I. Delaunay, D. Boichard, and S. Mattalia. 2003. A general approach for international genetic evaluations robust to inconsistencies of genetic trends in national evaluations. Interbull Bull. 30:101-111.

Ducrocq, V., and Z. Liu. 2009. Combining genomic and classical information in national BLUP evaluations. Interbull Bull. 40:172-177.

Falconer, D. S., and T. F. C. Mackay. 1996. Introduction to Quantitative Genetics. 4th ed. Longman, Essex, UK.

Gengler, N., T. Dusseldorf, G. R. Wiggans, J. R. Wright, and T. Druet. 2000. Joint estimation of variances and effects in the US Jersey type evaluation system. Interbull Bull. 26:34-39.

Hayes, B. J., P. J. Bowman, A. J. Chamberlain, and M. E. Goddard. 2009. Invited review: Genomic selection in dairy cattle: Progress and challenges. J. Dairy Sci. 92:433-443.

Interbull. 2017. International Bull Evaluation Service. Accessed Apr. 13, 2017. http://www.interbull.org/index.

MiX99 Development Team. 2015. MiX99: A software package for solving large mixed model equations. Natural Resources Institute Finland (Luke), Jokioinen, Finland. Accessed Apr. 15, 2017. http:// www.luke.fi/mix99.

Miglior, F., P. G. Sullivan, and B. J. van Doormaal. 2002. Preliminary analysis of Mendelian sampling terms for genetic evaluation validation. Interbull Bull. 29:183-187.

Misztal, I., A. Legarra, and I. Aguilar. 2014. Using recursion to compute the inverse of the genomic relationship matrix. J. Dairy Sci. 97:3943-3952.

Misztal, I., and G. R. Wiggans. 1988. Approximation of prediction error variance in large-scale animal models. J. Dairy Sci. 71(Suppl. 2):27-32

Patry, C., and V. Ducrocq. 2011a. Accounting for genomic pre-selection in national BLUP evaluations in dairy cattle. Genet. Sel. Evol. 43:30.

Patry, C., and V. Ducrocq. 2011b. Evidence of biases in genetic evaluations due to genomic preselection in dairy cattle. J. Dairy Sci. 94:1011-1020.

Patry, C., H. Jorjani, and V. Ducrocq. 2013. Effects of a national genomic preselection on the international genetic evaluations. J. Dairy Sci. 96:3272-3284

Sorensen, D. A., and B. W. Kennedy. 1984. Estimation of response to selection using least-squares and mixed model methodology. J. Anim. Sci. 58:1097-1106.

Taskinen, M., E. A. Mäntysaari, and I. Strandén. 2017. Single-step SNP-BLUP with on-the-fly imputed genotypes and residual polygenic effects. Genet. Sel. Evol. 49:36.

Tyrisevä, A.-M., W. F. Fikse, E. A. Mäntysaari, J. Jakobsen, G. P. Aamand, J. Dürr, and M. H. Lidauer. 2018. Validation of consistency of Mendelian sampling variance. J. Dairy Sci. 101:2187-2198. https://doi.org/10.3168/jds.2017-13255.

Tyrisevä, A.-M., E. A. Mäntysaari, J. Jakobsen, G. P. Aamand, J. Dürr, W. F. Fikse, and M. H. Lidauer. 2013. Detection of genomic preselection with Mendelian sampling variance test. Interbull Bull. 47:197-202.

Van Doormaal, B. J., G. J. Kistemaker, and P. G. Sullivan. 1999. Heterogeneous variances of Canadian bull EBVs over time. Interbull Bull. 22:141-148.

VanRaden, P. M. 2008. Efficient methods to compute genomic predictions. J. Dairy Sci. 91:4414-4423. https://doi.org/10.3168/jds .2007-0980.

VanRaden, P. M., and J. R. Wright. 2013. Measuring genomic preselection in theory and in practice. Interbull Bull. 47:147-150.

Vitezica, Z. G., I. Aguilar, I. Misztal, and A. Legarra. 2011. Bias in genomic predictions for populations under selection. Genet. Res. (Camb.) 93:357-366.

Weigel, K. A., G. Banos, and A. Sigurdsson. 1996. International sire evaluations and conversions in upgrading populations with changing means and variances. Interbull Bull. 14:21-25. 